\title{
VARIATIONAL PRINCIPLES FOR LINEAR INITIAL-VALUE PROBLEMS*
}

By M. E. GURTIN (Brown University)

Introduction. Most of the boundary-value problems of mathematical physics are characterized by variational principles which assert that a function $u$ satisfies such a problem if and only if a given functional is stationary at $u$. However, there does not seem to exist in the treatise literature any such variational characterization of the usual initial-value problems of interest. As a matter of fact Washizu [1] conjectured that in the case of the transient heat conduction problem such a principle is not possible since the problem is not self-adjoint. In this note, variational principles of the foregoing type are derived for the initial-value problems associated with the wave and heat conduction equations, thus proving Washizu's conjecture incorrect.

The method of deducing such variational principles consists of two steps. First we reduce the initial-value problem to an equivalent boundary-value problem by replacing the relevant partial differential equation with an equivalent integro-differential equation which contains the initial conditions implicitly. Second, we derive a variational principle for this reduced problem. The prime ingredient in the derivation of this variational principle is the use of convolutions.

The techniques employed in this paper may be used to generate variational principles for a large class of initial-value problems (e.g. the initial-value problem associated with the time-dependent Schrödinger equation in quantum mechanics). Variational principles of this type for linear elastodynamics were given previously [2].

1. Preliminary definitions. Notation. Henceforth $\bar{R}$ will denote a closed and bounded region in $n$-dimensional Euclidean space with the interior $R$ and the boundary $B$, while $x=\left(x_{1}, x_{2}, \cdots, x_{n}\right)$ is a generic point of $\bar{R}$. Moreover $\nabla$ is the usual $(n$ dimensional) gradient operator and $\nabla^{2}$ is the Laplacian operator. Throughout this paper we deal with real-valued functions of position $x$ and time $t$ whose $n+1$ dimensional domain of definition is the Cartesian product $\bar{R} \times[0, \infty)$ of the region $\bar{R}$ with the time interval $[0, \infty)$. We use the standard notation for the convolution of two such functions of position and time, i.e.

$$
u * v(x, t)=\int_{0}^{t} u(x, t-\tau) v(x, \tau) d \tau
$$

Moreover we define

$$
\nabla u * \nabla v=\sum_{k=1}^{n} \frac{\partial u}{\partial x_{k}} * \frac{\partial v}{\partial x_{k}} .
$$

The customary notation for the calculus of variations will be used throughout this paper. Thus let $\Phi(\cdot)$ be a functional defined on a subset $K$ of some function space and suppose $u$ and $\delta u$ belong to this space. Further, let

$$
u+\lambda \delta u \varepsilon K
$$

for every real number $\lambda$ and formally define the notation

\footnotetext{
*Received November 8, 1963.
} 


$$
\delta \Phi(u)=\left.\frac{d}{d \lambda} \Phi(u+\lambda \delta u)\right|_{\lambda=0} .
$$

We then write

$$
\delta \Phi(u)=0 \text { over } K
$$

if and only if (1.4) exists and equals zero for every choice of $\delta u$ consistent with (1.3).

In the interest of clarity we omit all hypotheses concerning continuity, differentiability, etc. It will be clear from the context what these ought to be. Thus a statement such as "all functions" actually means "all sufficiently smooth functions".

2. The wave problem. Consider the initial-value problem which consists in finding a solution $u$ on $\bar{R} \times[0, \infty)$ of the wave equation

$$
c^{2} \nabla^{2} u=\frac{\partial^{2} u}{\partial t^{2}}
$$

that meets the initial conditions

$$
u(x, 0)=u_{0}(x), \quad \frac{\partial u(x, 0)}{\partial t}=v_{0}(x), \quad x \varepsilon R,
$$

as well as the boundary condition

$$
u=U \quad \text { on } B \times(0, \infty) .
$$

Here $c$ is a real constant, while $u_{0}, v_{0}$, and $U$ are prescribed functions. This initial-value problem will be referred to as the wave problem.

In order to see the difficulties that arise in trying to deduce a variational characterization of this problem, recall the well-known variational principle for the wave equation. Suppose we are given a positive time $t_{0}$ and let $K_{t_{\mathrm{o}}}$ be the set of all functions $u$ which meet the boundary conditions $u=U$ on $B \times\left(0, t_{0}\right)$ as well as

$$
u(x, 0)=u_{0}(x), \quad u\left(x, t_{0}\right)=u_{t_{0}}(x), \quad x \varepsilon R,
$$

where $u_{t_{0}}$ is a prescribed function. Let $u \varepsilon K_{t_{0}}$. Then this principle asserts that

$$
\delta\left\{\frac{1}{2} \int_{0}^{t_{\bullet}} \int_{R}\left[c^{2} \nabla u \cdot \nabla u-\left(\frac{\partial u}{\partial t}\right)^{2}\right](x, t) d x d t\right\}=0 \text { over } K_{t 。}
$$

if and only if $u$ meets the wave equation (2.1) on $R \times\left(0, t_{0}\right)$. This variational principle is of the type represented by Hamilton's principle in classical dynamics. It is clearly inapplicable to the wave problem since it fails to take into account the initial velocity distribution $v_{0}(x)$ and presupposes the knowledge of the function $u$ at a later time $t_{0}$-an item of information not available in advance.

Since the major difficulty in trying to develop a variational principle for the wave problem appears to center around the initial conditions it would seem advisable to develop an alternative formulation of this problem which does not contain the initial conditions explicitly. To this end proceed formally and take the Laplace transform of the wave equation (2.1). This result together with the initial conditions (2.2) yields

$$
c^{2} \nabla^{2} w(x, s)-s^{2} w(x, s)=-s u_{0}(x)-v_{0}(x),
$$

where $w(x, s)$ is the Laplace transform (with respect to $t$ ) of $u(x, t)$. Now divide (2.6) through by $s^{2}$, take the inverse Laplace transform, and use the fact that multiplication 
in the "transform domain" corresponds to convolution in the "time domain" to arrive at

$$
g * \nabla^{2} u-u=f
$$

where

$$
\begin{gathered}
g(x, t)=c^{2} t, \\
f(x, t)=-u_{0}(x)-t v_{0}(x) .
\end{gathered}
$$

Thus we are led to the following lemma.

Lemma. A function $u$ on $R \times[0, \infty)$ meets the wave equation (2.1) and the initial conditions (2.2) if and only if $u$ satisfies (2.7), (2.8).

We now give a proof of this lemma which does not require the use of the Laplace transform. Suppose (2.1), (2.2) hold. Then (2.8) implies

$$
\begin{aligned}
g * \nabla^{2} u(x, t) & =\int_{0}^{t}(t-\tau) \frac{\partial^{2} u(x, \tau)}{\partial \tau^{2}} d \tau \\
& =u(x, t)-t v_{0}(x)-u_{0}(x) \\
& =u(x, t)+f(x, t) .
\end{aligned}
$$

Conversely, suppose (2.7) holds. Then, by reversing the foregoing argument it is easily verified that $u$ meets (2.1), (2.2). Thus the wave problem is equivalent to the reduced wave problem which consists in finding a solution $u$ of the integro-differential equation (2.7) which meets the boundary condition (2.3). This fact enables us to prove the following principle.

Variational Principle for the Wave Problem. Let $K$ be the set of all functions $u$ which meet the boundary condition (2.3). For each $t \varepsilon[0, \infty)$ define the functional $\Lambda_{t}(\cdot)$ on $K$ through

$$
\Lambda_{t}(u)=\int_{R}[u * u+g * \nabla u * \nabla u+2 f * u](x, t) d x,
$$

where $g$ and $f$ are given by (2.8). Then

$$
\delta \Lambda_{t}(u)=0 \quad \text { over } K \quad(0 \leq t<\infty)
$$

at a particular function $u$ if and only if $u$ is a solution of the wave problem (2.1), (2.2), (2.3)

Proof. Let $u+\lambda \delta u \varepsilon K$ for every real number $\lambda$ from which it follows that

$$
\delta u=0 \text { on } B \times(0, \infty) .
$$

Therefore (2.10), (2.12), (1.2), the divergence theorem, and the associativity and commutativity of the convolution (see for instance [3]) imply

$$
\delta \Lambda_{t}(u)=2 \int_{R}\left[u-g * \nabla^{2} u+f\right] * \delta u(x, t) d x \quad(0 \leq t<\infty) .
$$

First suppose $u$ is a solution of the wave problem. Then by the lemma $u$ meets (2.7) and hence (2.11) holds. Conversely suppose $u$ satisfies (2.11). Then (2.13) implies

$$
\int_{R} \int_{0}^{t}\left[u-g * \nabla^{2} u-f\right](x, t-\tau) \delta u(x, \tau) d \tau d x=0 \quad(0 \leq t<\infty)
$$


for every $\delta u$ which vanishes on $B \times(0, \infty)$. But this fact together with the fundamental lemma of the calculus of variations implies the validity of (2.7). Thus and by virtue of the previous lemma $u$ meets the wave equation (2.1) as well as the initial conditions (2.2). Moreover, since $u \varepsilon K$, the boundary condition (2.3) also holds. This completes the proof.

3. The heat-conduction problem. We turn now to the heat conduction problem which consists in finding a solution $u$ on $\bar{R} \times[0, \infty)$ of the heat equation

$$
\alpha^{2} \nabla^{2} u=\frac{\partial u}{\partial t},
$$

which meets the initial condition

$$
u(x, 0)=u_{0}(x), \quad x \varepsilon R,
$$

together with the boundary condition

$$
u=U \text { on } B \times(0, \infty) .
$$

Here $\alpha$ is a real constant, while $u_{0}$ and $U$ are prescribed functions.

We now proceed in the same manner as we did in the case of the wave problem and first prove the following lemma.

Lemma. A function $u$ on $R \times[0, \infty)$ satisfies the heat equation (3.1) and the initial condition (3.2) if and only if $u$ meets

$$
q * \nabla^{2} u-u=p,
$$

where

$$
\begin{aligned}
& q(x, t)=\alpha^{2}=\text { constant }, \\
& p(x, t)=-u_{0}(x) .
\end{aligned}
$$

The proof of this lemma follows immediately from the fact that (3.4), by virtue of (3.5), is equivalent to

$$
\int_{0}^{t} \alpha^{2} \nabla^{2} u(x, \tau) d \tau=u(x, t)-u_{0}(x) .
$$

If we compare (3.4) with (2.7) we are at once led to the following principle.

Variational Principle for the Heat Conduction Problem. Let $K$ be the set of all functions $u$ which meet the boundary condition (3.3). For each $t \varepsilon[0, \infty)$ define the functional $\Omega_{\mathfrak{t}}(\cdot)$ on $K$ through

$$
\Omega_{t}(u)=\int_{R}[u * u+q * \nabla u * \nabla u+2 p * u](x, t) d x,
$$

where $q$ and $p$ are given by (3.5). Then

$$
\delta \Omega_{t}(u)=0 \quad \text { over } K \quad(0 \leq t<\infty)
$$

at a particular function $u$ if and only if $u$ is a solution of the heat conduction problem (3.1), (3.2), (3.3).

The proof of this theorem is exactly the same as the proof of the variational principle for the wave problem. 
4. Generalizations. The results for the wave equation can easily be generalized to include the inhomogeneous wave equation

$$
c^{2} \nabla^{2} u=\frac{\partial^{2} u}{\partial t^{2}}+F
$$

together with the mixed boundary-conditions

$$
u=U \quad \text { on } B_{1} \times(0, \infty), \quad \frac{\partial u}{\partial n}=V \quad \text { on } B_{2} \times(0, \infty)
$$

and the initial conditions (2.2). Indeed, all one has to do is add the terms

$$
\left(2 / c^{2} \int_{R} g * F * u(x, t) d x-2 \int_{B,} g * V * u(x, t) d x\right.
$$

to the right hand side of (2.10) and require that $K$ be the set of all functions $u$ which satisfy $u=U$ on $B_{1} \times(0, \infty)$. The analogous assertion applies to the heat conduction problem.

\section{ReFerences}

1. K. Washizu, Variational principles in continuum mechanics, Rept. No. 62-2, Dept. Aero. Engrg., University of Washington, 1962

2. M. E. Gurtin, Variational principles for linear elastodynamics, Archive Ratl. Mech. Anal. 16, 1, 1964

3. J. Mikusinski, Operational calculus, Pergamon Press, New York, 1959

\section{WAVE OPERATORS AND ABSOLUTELY CONTINUOUS SPECTRA*}

By C. R. PUTNAM (Purdue University)

1. On a Hilbert space $\mathfrak{S}$ of elements $f, g, \cdots$, with inner product $(f, g)$, let $\{E(\lambda)\}$, $-\infty<\lambda<\infty$, denote a one-dimensional spectral family. If $\mathfrak{S}_{a}$ denotes the Hilbert space spanned by the set of elements $f$ for which $\|E(\lambda) f\|^{2}$ is a absolutely continuous function of $\lambda$, then $\mathfrak{S}_{a}$ reduces the family $\{E(\lambda)\}$ and will be called the absolutely continuous part of $\mathfrak{S}$ determined by the spectral family $\{E(\lambda)\}$; see Halmos [1], p. 104, Kato [5], p. 240, Kuroda [6], p. 436. In the case of a self-adjoint operator $H$ or a unitary operator $U$, there exists in each instance a spectral family $\{E(\lambda)\}$ for which

$$
H=\int_{-\infty}^{\infty} \lambda d E(\lambda) \text { or } U=\int_{0}^{2 \pi} e^{i \lambda} d E(\lambda) .
$$

The restriction of $H$ (or $U$ ) to the corresponding space $\mathfrak{S}_{a}$ will be called the absolutely continuous part of $H$ (or $U$ ). The operator $H$ or $U$ will be called absolutely continuous on a subspace $\mathfrak{M}$ of $\mathfrak{S}$ if $\mathfrak{M} \subset \mathfrak{S}_{a}$.

If $\mathfrak{M}$ is a subspace its orthogonal complement in $\mathfrak{S}$ will be denoted by $\mathfrak{M}^{\perp}$. The space $\mathfrak{S}_{\mathfrak{l}_{1}}=\mathfrak{S}_{a}^{\perp}$ is spanned by the singular elements. Thus, if $f$ is in $\mathfrak{S}_{s}$ and if $f \neq 0$, then the absolutely continuous part of the monotone function $\|E(\lambda) f\|^{2}$ in the Lebesgue

${ }^{*}$ Received December 2, 1963. This work was supported by the National Science Foundation research grant NSF-GP1665. 